Commun. Korean Math. Soc. 25 (2010), No. 1, pp. 59-68

DOI 10.4134/CKMS.2010.25.1.059

\title{
COMMON FIXED POINT THEOREM OF SEMI-COMPATIBLE MAPS ON INTUITIONISTIC FUZZY METRIC SPACE
}

\author{
Jong SEO PARK
}

\begin{abstract}
In this paper, we prove common fixed point theorems for semi-compatible mappings on intuitionistic fuzzy metric space with different some conditions of Park and Kim [10]. This research extended and generalized the results of Singh and Chauhan [14].
\end{abstract}

\section{Introduction}

The concept of fuzzy set was developed extensively by many authors and used in various fields. Several authors have defined fuzzy metric space ([5] etc.) with various methods to use this concept in analysis. Jungck ([3], [4]) researched the more generalized concept compatibility than commutativity and weak commutativity in metric space and proved common fixed point theorems, and Singh and Chauhan [14] introduced the concept of compatibility in fuzzy metric space and studied common fixed point theorems for four compatible mappings.

Recently, Park et. al. [7] defined the upgraded intuitionistic fuzzy metric space and Park et. al. ([8], [9], [11], [12]) studied several theories in this space. Also, Park and Kim [10] proved common fixed point theorem for self maps in intuitionistic fuzzy metric space.

In this paper, we prove common fixed point theorems for semi-compatible mappings on intuitionistic fuzzy metric space with different some conditions of Park and Kim [10]. This research extended and generalized the results of Singh and Chauhan [14].

\section{Preliminaries}

We give some definitions and properties of intuitionistic fuzzy metric space. Throughout this paper, $\mathbb{N}$ will denote the set of all positive integers.

Received October 1, 2008.

2000 Mathematics Subject Classification. 46S40, 47H10, 47S40.

Key words and phrases. intuitionistic fuzzy metric space, Cauchy sequence, complete, compatible map, semi-compatible map. 
Let us recall (see [13]) that a continuous $t$-norm is a binary operation $*$ : $[0,1] \times[0,1] \rightarrow[0,1]$ which satisfies the following conditions:
(a) $*$ is commutative and associative;
(b) $*$ is continuous;
(c) $a * 1=a$ for all $a \in[0,1]$;
(d) $a * b \leq c * d$ whenever $a \leq c$ and $b \leq d(a, b, c, d \in[0,1])$.

Similarly, a continuous $t$-conorm is a binary operation $\diamond:[0,1] \times[0,1] \rightarrow[0,1]$ which satisfies the following conditions:

(a) $\diamond$ is commutative and associative;

(b) $\diamond$ is continuous;

(c) $a \diamond 0=a$ for all $a \in[0,1]$;

(d) $a \diamond b \geq c \diamond d$ whenever $a \leq c$ and $b \leq d(a, b, c, d \in[0,1])$.

Also, let us recall (see [6]) that the following conditions are satisfied:

(a) For any $r_{1}, r_{2} \in(0,1)$ with $r_{1}>r_{2}$, there exist $r_{3}, r_{4} \in(0,1)$ such that $r_{1} * r_{3} \geq r_{2}$ and $r_{4} \diamond r_{2} \leq r_{1}$;

(b) For any $r_{5} \in(0,1)$, there exist $r_{6}, r_{7} \in(0,1)$ such that $r_{6} * r_{6} \geq r_{5}$ and $r_{7} \diamond r_{7} \leq r_{5}$.

Definition $2.1([7])$. The 5 -tuple $(X, M, N, *, \diamond)$ is said to be an intuitionistic fuzzy metric space if $X$ is an arbitrary set, $*$ is a continuous $t$-norm, $\diamond$ is a continuous $t$-conorm and $M, N$ are fuzzy sets on $X^{2} \times(0, \infty)$ satisfying the following conditions; for all $x, y, z \in X$, such that
(a) $M(x, y, t)>0$,
(b) $M(x, y, t)=1 \Longleftrightarrow x=y$
(c) $M(x, y, t)=M(y, x, t)$,
(d) $M(x, y, t) * M(y, z, s) \leq M(x, z, t+s)$,
(e) $M(x, y, \cdot):(0, \infty) \rightarrow(0,1]$ is continuous,
(f) $N(x, y, t)>0$
(g) $N(x, y, t)=0 \Longleftrightarrow x=y$,
(h) $N(x, y, t)=N(y, x, t)$,
(i) $N(x, y, t) \diamond N(y, z, s) \geq N(x, z, t+s)$,
(j) $N(x, y, \cdot):(0, \infty) \rightarrow(0,1]$ is continuous.

Note that $(M, N)$ is called an intuitionistic fuzzy metric on $X$. The functions $M(x, y, t)$ and $N(x, y, t)$ denote the degree of nearness and the degree of nonnearness between $x$ and $y$ with respect to $t$, respectively.

Definition 2.2 ([12]). Let $X$ be an intuitionistic fuzzy metric space. Then

(a) A sequence $\left\{x_{n}\right\} \subset X$ is convergent to $x$ in $X$ if and only if for each $\epsilon>0, t>0$, there exists $n_{0} \in \mathbb{N}$ such that $M\left(x_{n}, x, t\right)>1-\epsilon, N\left(x_{n}, x, t\right)<\epsilon$ for all $n \geq n_{0}$.

(b) A sequence $\left\{x_{n}\right\} \subset X$ is called Cauchy sequence if and only if for each $\epsilon>0, t>0$, there exists $n_{0} \in \mathbb{N}$ such that $M\left(x_{n}, x_{m}, t\right)>1-\epsilon, N\left(x_{n}, x_{m}, t\right)<$ $\epsilon$ for all $n, m \geq n_{0}$.

(c) $X$ is complete if every Cauchy sequence in $X$ is convergent. 
Definition 2.3 ([10]). Let $A, B$ be mappings from intuitionistic fuzzy metric space $X$ into itself.

(a) $(A, B)$ are said to be compatible if and only if

$$
\lim _{n \rightarrow \infty} M\left(A B x_{n}, B A x_{n}, t\right)=1, \lim _{n \rightarrow \infty} N\left(A B x_{n}, B A x_{n}, t\right)=0
$$

for all $t>0$, whenever $\left\{x_{n}\right\} \subset X$ such that $\lim _{n \rightarrow \infty} A x_{n}=\lim _{n \rightarrow \infty} B x_{n}=x$ for some $x \in X$.

(b) $(A, B)$ are said to be semi-compatible if and only if

$$
\lim _{n \rightarrow \infty} M\left(A B x_{n}, B x, t\right)=1, \lim _{n \rightarrow \infty} N\left(A B x_{n}, B x, t\right)=0
$$

for all $t>0$, whenever $\left\{x_{n}\right\} \subset X$ such that $\lim _{n \rightarrow \infty} A x_{n}=\lim _{n \rightarrow \infty} B x_{n}=x$ for some $x \in X$.

Lemma 2.4 ([10]). Let $A, B$ be self mappings on intuitionistic fuzzy metric space $X$. If $B$ is continuous, then $(A, B)$ is semi-compatible if and only if $(A, B)$ is compatible.

\section{Main results}

Theorem 3.1. Let $A, B, S$ and $T$ be self maps of complete intuitionistic fuzzy metric space $X$ with $t$-norm $*$ and $t$-conorm $\diamond$ defined by $\alpha * \beta=\min \{\alpha, \beta\}$ and $\alpha \diamond \beta=\max \{\alpha, \beta\}, \alpha, \beta \in[0,1]$, satisfying

(a) $(A, S)$ and $(B, T)$ are semi-compatible pairs of maps,

(b) $S$ and $T$ are continuous,

(c) $A^{a}(X) \subset T^{t}(X), B^{b}(X) \subset S^{s}(X)$,

(d)

$$
\begin{aligned}
& M\left(A^{a} x, B^{b} y, k t\right) \\
\geq \quad & \min \left\{M\left(S^{s} x, T^{t} y, t\right), M\left(A^{a} x, S^{s} x, t\right), M\left(B^{b} y, T^{t} y, t\right), M\left(B^{b} y, S^{s} x, 2 t\right),\right. \\
& \left.M\left(A^{a} x, T^{t} y, t\right)\right\}, \\
& N\left(A^{a} x, B^{b} y, k t\right) \\
\leq & \max \left\{N\left(S^{s} x, T^{t} y, t\right), N\left(A^{a} x, S^{s} x, t\right), N\left(B^{b} y, T^{t} y, t\right), N\left(B^{b} y, S^{s} x, 2 t\right),\right. \\
& \left.N\left(A^{a} x, T^{t} y, t\right)\right\} .
\end{aligned}
$$

(e) $\lim _{t \rightarrow \infty} M(x, y, t)=1, \lim _{t \rightarrow \infty} N(x, y, t)=0$ for all $x, y \in X, t>0$ and $a, b, s, t \in \mathbb{N}$.

Then $A, B, S$ and $T$ have a unique common fixed point in $X$.

Proof. Let $x_{0}$ be an arbitrary point in $X$, we can inductively construct a sequence $\left\{y_{n}\right\} \subset X$ such that $y_{2 n-1}=T^{t} x_{2 n-1}=A^{a} x_{2 n-2}, y_{2 n}=S^{s} x_{2 n}=$ $B^{b} x_{2 n-1}$ for $n=1,2,3, \ldots$

First, we prove that $\left\{y_{n}\right\}$ is Cauchy sequence. From (d), we have

$$
\begin{aligned}
& M\left(y_{2 n+1}, y_{2 n+2}, k t\right) \\
= & M\left(A^{a} x_{2 n}, B^{b} x_{2 n+1}, k t\right)
\end{aligned}
$$




$$
\begin{aligned}
\geq & \min \left\{M\left(S^{s} x_{2 n}, T^{t} x_{2 n+1}, t\right), M\left(A^{a} x_{2 n}, S^{s} x_{2 n}, t\right), M\left(B^{b} x_{2 n+1}, T^{t} x_{2 n+1}, t\right)\right. \\
& \left.M\left(B^{b} x_{2 n+1}, S^{s} x_{2 n}, 2 t\right), M\left(A^{a} x_{2 n}, T^{t} x_{2 n+1}, t\right)\right\} \\
\geq & \min \left\{M\left(y_{2 n}, y_{2 n+1}, t\right), M\left(y_{2 n+1}, y_{2 n}, t\right), M\left(y_{2 n+2}, y_{2 n+1}, t\right),\right. \\
& \left.\quad M\left(y_{2 n+2}, y_{2 n}, 2 t\right), M\left(y_{2 n+1}, y_{2 n+1} t\right)\right\} \\
\geq & \min \left\{M\left(y_{2 n}, y_{2 n+1}, t\right), M\left(y_{2 n+2}, y_{2 n+1}, t\right), 1\right\} \\
& N\left(y_{2 n+1}, y_{2 n+2}, k t\right) \\
= & N\left(A^{a} x_{2 n}, B^{b} x_{2 n+1}, k t\right) \\
\leq & \max \left\{N\left(S^{s} x_{2 n}, T^{t} x_{2 n+1}, t\right), N\left(A^{a} x_{2 n}, S^{s} x_{2 n}, t\right), N\left(B^{b} x_{2 n+1}, T^{t} x_{2 n+1}, t\right)\right. \\
& \left.\quad N\left(B^{b} x_{2 n+1}, S^{s} x_{2 n}, 2 t\right), N\left(A^{a} x_{2 n}, T^{t} x_{2 n+1}, t\right)\right\} \\
\leq & \max \left\{N\left(y_{2 n}, y_{2 n+1}, t\right), N\left(y_{2 n+1}, y_{2 n}, t\right), N\left(y_{2 n+2}, y_{2 n+1}, t\right),\right. \\
& \left.\quad N\left(y_{2 n+2}, y_{2 n}, 2 t\right), N\left(y_{2 n+1}, y_{2 n+1} t\right)\right\} \\
\leq & \max \left\{N\left(y_{2 n}, y_{2 n+1}, t\right), N\left(y_{2 n+2}, y_{2 n+1}, t\right), 0\right\}
\end{aligned}
$$

which implies

$M\left(y_{2 n+1}, y_{2 n+2}, k t\right) \geq M\left(y_{2 n}, y_{2 n+1}, t\right), N\left(y_{2 n+1}, y_{2 n+2}, k t\right) \leq N\left(y_{2 n}, y_{2 n+1}, t\right)$.

Generally,

$$
M\left(y_{n}, y_{n+1}, k t\right) \geq M\left(y_{n-1}, y_{n}, t\right), \quad N\left(y_{n}, y_{n+1}, k t\right) \leq M\left(y_{n-1}, y_{n}, t\right) .
$$

Therefore

$$
\begin{aligned}
M\left(y_{n}, y_{n+1}, t\right) & \geq M\left(y_{n-1}, y_{n}, \frac{t}{k}\right) \\
& \geq \cdots \cdots \ldots \\
& \geq M\left(y_{0}, y_{1}, \frac{t}{k^{n}}\right) \\
& \rightarrow 1 \text { as } n \rightarrow \infty, \\
N\left(y_{n}, y_{n+1}, t\right) & \leq N\left(y_{n-1}, y_{n}, \frac{t}{k}\right) \\
& \leq \cdots \ldots \ldots\left(y_{0}, y_{1}, \frac{t}{k^{n}}\right) \\
& \leq N \text { as } n \rightarrow \infty .
\end{aligned}
$$

Hence for $t>0$ and $\epsilon \in(0,1)$, we can choose $n_{0} \in \mathbb{N}$ such that

$$
M\left(y_{n}, y_{n+1}, t\right)>1-\epsilon, \quad N\left(y_{n}, y_{n+1}, t\right)<\epsilon
$$

for all $n \geq n_{0}$.

Suppose that for $m$,

$$
M\left(y_{n}, y_{n+m}, t\right)>1-\epsilon, \quad N\left(y_{n}, y_{n+m}, t\right)<\epsilon
$$


for all $n \geq n_{0}$ and for every $m \in \mathbb{N}$. Then

$$
\begin{aligned}
& M\left(y_{n}, y_{n+m+1}, t\right) \\
\geq & \min \left\{M\left(y_{n}, y_{n+m}, \frac{t}{2}\right), M\left(y_{n+m}, y_{n+m+1}, \frac{t}{2}\right)\right\} \\
> & 1-\epsilon \\
& N\left(y_{n}, y_{n+m+1}, t\right) \\
\leq & \max \left\{N\left(y_{n}, y_{n+m}, \frac{t}{2}\right), N\left(y_{n+m}, y_{n+m+1}, \frac{t}{2}\right)\right\} \\
< & \epsilon .
\end{aligned}
$$

Therefore $\left\{y_{n}\right\} \subset X$ is a Cauchy sequence.

Second, we prove that $A^{a}, B^{b}, S^{s}$ and $T^{t}$ have a unique common fixed point. Since $\left\{y_{n}\right\}$ converges to some point $x$ from completeness of $X, A^{a} x_{2 n} \rightarrow x$, $S^{s} x_{2 n} \rightarrow x, B^{b} x_{2 n-1} \rightarrow x$ and $T^{t} x_{2 n-1} \rightarrow x$. Since $S$ is continuous, hence $S^{s}\left(A^{a} x_{2 n}\right) \rightarrow S^{s}(x)$. Thus for $t>0$ and $\epsilon \in(0,1)$, there exists an $n_{0} \in \mathbb{N}$ such that

$$
M\left(S^{s}\left(A^{a} x_{2 n}\right), S^{s}(x), \frac{t}{2}\right)>1-\epsilon, \quad N\left(S^{s}\left(A^{a} x_{2 n}\right), S^{s}(x), \frac{t}{2}\right)<\epsilon
$$

for all $n \geq n_{0}$. Also, since $(A, S)$ and $(B, T)$ are semi-compatible pairs, by Lemma $2.4,(A, S)$ and $(B, T)$ are compatible pairs. Therefore $\left(A^{a}, S^{s}\right)$ and $\left(B^{b}, T^{t}\right)$ are compatible pairs for all $a, b, s, t \in \mathbb{N}$. From (a), we have

$$
\begin{aligned}
& \lim _{n \rightarrow \infty} M\left(A^{a}\left(S^{s} x_{2 n}\right), S^{s}\left(A^{a} x_{2 n}\right), \frac{t}{2}\right)=1, \\
& \lim _{n \rightarrow \infty} N\left(A^{a}\left(S^{s} x_{2 n}\right), S^{s}\left(A^{a} x_{2 n}\right), \frac{t}{2}\right)=0 .
\end{aligned}
$$

Hence

$$
\begin{aligned}
& M\left(S^{s}\left(A^{a} x_{2 n}\right), S^{s}(x), t\right) \\
\geq & \min \left\{M\left(A^{a}\left(S^{s} x_{2 n}\right), S^{s}\left(A^{a} x_{2 n}\right), \frac{t}{2}\right), M\left(S^{s} A^{a}\left(x_{2 n}\right), S^{s} x, \frac{t}{2}\right)\right\} \\
> & 1-\epsilon \\
& N\left(S^{s}\left(A^{a} x_{2 n}\right), S^{s}(x), t\right) \\
\leq & \max \left\{M\left(A^{a}\left(S^{s} x_{2 n}\right), S^{s}\left(A^{a} x_{2 n}\right), \frac{t}{2}\right), M\left(S^{s} A^{a}\left(x_{2 n}\right), S^{s} x, \frac{t}{2}\right)\right\} \\
< & \epsilon
\end{aligned}
$$

for all $n \geq n_{0}$. Therefore $\lim _{n \rightarrow \infty} A^{a} S^{s} x_{2 n}=S^{s} x$. Also, since $\lim _{n \rightarrow \infty} B^{b} x_{2 n-1}$ $=x$ and $T$ is continuous, $\lim _{n \rightarrow \infty} T^{t}\left(B^{b} x_{2 n-1}\right)=T^{t} x$. Thus for $t>0$ and 
$\epsilon \in(0,1)$, there exists an $n_{0} \in \mathbb{N}$ such that

$$
M\left(T^{t}\left(B^{b} x_{2 n-1}\right), T^{t}(x), \frac{t}{2}\right)>1-\epsilon, \quad N\left(T^{t}\left(B^{b} x_{2 n-1}\right), T^{t}(x), \frac{t}{2}\right)<\epsilon
$$

for all $n \geq n_{0}$.

From (a), we have

$$
\begin{aligned}
& \lim _{n \rightarrow \infty} M\left(B^{b}\left(T^{t} x_{2 n-1}\right), T^{t}\left(B^{b} x_{2 n-1}\right), \frac{t}{2}\right)=1, \\
& \lim _{n \rightarrow \infty} N\left(B^{b}\left(T^{t} x_{2 n-1}\right), T^{t}\left(B^{b} x_{2 n-1}\right), \frac{t}{2}\right)=0 .
\end{aligned}
$$

Hence

$$
\begin{aligned}
& M\left(B^{b}\left(T^{t} x_{2 n-1}\right), T^{t} x, t\right) \\
\geq & \min \left\{M\left(B^{b}\left(T^{t} x_{2 n-1}\right), T^{t}\left(B^{b} x_{2 n-1}\right), \frac{t}{2}\right), M\left(T^{t}\left(B^{b} x_{2 n-1}\right), T^{t} x, t\right)\right\} \\
\geq & 1-\epsilon \\
& N\left(B^{b}\left(T^{t} x_{2 n-1}\right), T^{t} x, t\right) \\
\leq & \max \left\{N\left(B^{b}\left(T^{t} x_{2 n-1}\right), T^{t}\left(B^{b} x_{2 n-1}\right), \frac{t}{2}\right), N\left(T^{t}\left(B^{b} x_{2 n-1}\right), T^{t} x, t\right)\right\} \\
\leq & \epsilon
\end{aligned}
$$

for all $n \geq n_{0}$. Therefore $\lim _{n \rightarrow \infty} B^{b}\left(T^{t} x_{2 n-1}\right)=T^{t} x$.

Using (d), we have

$$
\begin{aligned}
& M\left(A^{a}\left(S^{s} x_{2 n}\right), B^{b}\left(T^{t} x_{2 n-1}\right), k t\right) \\
\geq \quad \min & M\left(S^{s}\left(S^{s} x_{2 n}\right), T^{t}\left(T^{t} x_{2 n-1}\right), t\right), M\left(A^{a}\left(S^{s} x_{2 n}\right), S^{s}\left(S^{s} x_{2 n}\right), t\right), \\
& M\left(B^{b}\left(T^{t} x_{2 n-1}\right), T^{t}\left(T^{t} x_{2 n-1}\right), t\right), M\left(B^{b}\left(T^{t} x_{2 n-1}\right), S^{s}\left(S^{s} x_{2 n}\right), 2 t\right), \\
& \left.M\left(A^{a}\left(S^{s} x_{2 n}\right), T^{t}\left(T^{t} x_{2 n-1}\right), t\right)\right\}, \\
& N\left(A^{a}\left(S^{s} x_{2 n}\right), B^{b}\left(T^{t} x_{2 n-1}\right), k t\right) \\
\leq \quad & \max \left\{N\left(S^{s}\left(S^{s} x_{2 n}\right), T^{t}\left(T^{t} x_{2 n-1}\right), t\right), N\left(A^{a}\left(S^{s} x_{2 n}\right), S^{s}\left(S^{s} x_{2 n}\right), t\right),\right. \\
& N\left(B^{b}\left(T^{t} x_{2 n-1}\right), T^{t}\left(T^{t} x_{2 n-1}\right), t\right), N\left(B^{b}\left(T^{t} x_{2 n-1}\right), S^{s}\left(S^{s} x_{2 n}\right), 2 t\right), \\
& \left.N\left(A^{a}\left(S^{s} x_{2 n}\right), T^{t}\left(T^{t} x_{2 n-1}\right), t\right)\right\} .
\end{aligned}
$$

Taking the limit as $n \rightarrow \infty$, and using above results,

$$
M\left(S^{s} x, T^{t} x, k t\right) \geq M\left(S^{s} x, T^{t} x, t\right), \quad N\left(S^{s} x, T^{t} x, k t\right) \leq N\left(S^{s} x, T^{t} x, t\right)
$$

which implies $S^{s} x=T^{t} x$.

Now,

$$
\begin{aligned}
& M\left(A^{a} x, B^{b}\left(T^{t} x_{2 n-1}\right), k t\right) \\
\geq \min \{ & M\left(S^{s} x, T^{t}\left(T^{t} x_{2 n-1}\right), t\right), M\left(A^{a} x, S^{s} x, t\right), M\left(B^{b}\left(T^{t} x_{2 n-1}\right),\right. \\
& \left.T^{t}\left(T^{t} x_{2 n-1}\right), t\right), M\left(B^{b}\left(T^{t} x_{2 n-1}\right), S^{s}\left(S^{s} x\right), 2 t\right),
\end{aligned}
$$




$$
\begin{aligned}
& \left.M\left(A^{a} x, T^{t}\left(T^{t} x_{2 n-1}\right), t\right)\right\}, \\
N\left(A^{a} x, B^{b}\left(T^{t} x_{2 n-1}\right), k t\right) & \\
\leq \max \{ & N\left(S^{s} x, T^{t}\left(T^{t} x_{2 n-1}\right), t\right), N\left(A^{a} x, S^{s} x, t\right), N\left(B^{b}\left(T^{t} x_{2 n-1}\right),\right. \\
& \left.T^{t}\left(T^{t} x_{2 n-1}\right), t\right), N\left(B^{b}\left(T^{t} x_{2 n-1}\right), S^{s}\left(S^{s} x\right), 2 t\right), \\
& \left.N\left(A^{a} x, T^{t}\left(T^{t} x_{2 n-1}\right), t\right)\right\} .
\end{aligned}
$$

Taking the limit as $n \rightarrow \infty$, and using above results,

$$
M\left(A^{a} x, T^{t} x, k t\right) \geq M\left(A^{a} x, T^{t} x, t\right), \quad N\left(A^{a} x, T^{t} x, k t\right) \leq N\left(A^{a} x, T^{t} x, t\right)
$$

which implies $A^{a} x=T^{t} x$. Also, since

$$
M\left(A^{a} x, B^{b} x, k t\right) \geq M\left(A^{a} x, B^{b} x, t\right), \quad N\left(A^{a} x, B^{b} x, k t\right) \leq N\left(A^{a} x, B^{b} x, t\right) .
$$

Hence $A^{a} x=B^{b} x$. Therefore $A^{a} x=B^{b} x=S^{s} x=T^{t} x$. Furthermore, since

$$
\begin{aligned}
& M\left(A^{a} x_{2 n}, B^{b} x, k t\right) \\
\geq \quad & \min \left\{M\left(S^{s} x_{2 n}, T^{t} x, t\right), M\left(A^{a} x_{2 n}, S^{s} x_{2 n}, t\right), M\left(B^{b} x, T^{t} x, t\right),\right. \\
& \left.M\left(B^{b} x, S^{s} x_{2 n}, 2 t\right), M\left(A^{a} x_{2 n}, T^{t} x, t\right)\right\}, \\
& N\left(A^{a} x_{2 n}, B^{b} x, k t\right) \\
\leq & \max \left\{N\left(S^{s} x_{2 n}, T^{t} x, t\right), N\left(A^{a} x_{2 n}, S^{s} x_{2 n}, t\right), N\left(B^{b} x, T^{t} x, t\right),\right. \\
& \left.N\left(B^{b} x, S^{s} x_{2 n}, 2 t\right), N\left(A^{a} x_{2 n}, T^{t} x, t\right)\right\} .
\end{aligned}
$$

Taking the limit as $n \rightarrow \infty$,

$$
M\left(x, B^{b} x, k t\right) \geq M\left(x, B^{b} x, t\right), \quad N\left(x, B^{b} x, k t\right) \leq N\left(x, B^{b} x, t\right)
$$

which implies $x=B^{b} x$. Therefore $x=B^{b} x=A^{a} x=S^{s} x=T^{t} x$. That is, $x$ is a common fixed point of $A^{a}, B^{b}, S^{s}$ and $T^{t}$. Let $z$ be another common fixed point of maps. Then

$$
\begin{aligned}
& M\left(A^{a} x, B^{b} z, k t\right) \\
\geq & \min \left\{M\left(S^{s} x, T^{t} z, t\right), M\left(A^{a} x, S^{s} x, t\right), M\left(B^{b} z, T^{t} z, t\right),\right. \\
& \left.M\left(B^{b} z, S^{s} z, 2 t\right), M\left(A^{a} x, T^{t} z, t\right)\right\} \\
\geq \quad & \min \{M(x, z, t), M(x, x, t), M(z, z, t), \\
& M(z, z, 2 t), M(x, z, t)\} \\
\geq \quad & M(x, z, t), \\
& N\left(A^{a} x, B^{b} z, k t\right) \\
\leq \quad & \max \left\{N\left(S^{s} x, T^{t} z, t\right), N\left(A^{a} x, S^{s} x, t\right), N\left(B^{b} z, T^{t} z, t\right),\right. \\
& \left.N\left(B^{b} z, S^{s} z, 2 t\right), N\left(A^{a} x, T^{t} z, t\right)\right\} \\
\leq & \max \{N(x, z, t), N(x, x, t), N(z, z, t), \\
& N(z, z, 2 t), N(x, z, t)\} \\
\leq \quad & N(x, z, t) .
\end{aligned}
$$

Hence $x$ is a unique common fixed point of maps. 
Third, we prove that this point $x$ is a common fixed point of $A, B, S$ and $T$. Since $A x=A\left(A^{a} x\right)=A^{a}(A x)$ and $A x=A\left(S^{s} x\right)=S^{s}(A x)$ from (a), hence $A x$ is a common fixed point of $A^{a}$ and $S^{s}$. Also, since $B x=B\left(B^{b} x\right)=B^{b}(B x)$ and $B x=B\left(T^{t} x\right)=T^{t}(B x)$ from (a), hence $B x$ is a common fixed point of $B^{b}$ and $T^{t}$. Now, letting $x=A x$ and $y=B x$ in (d), we have

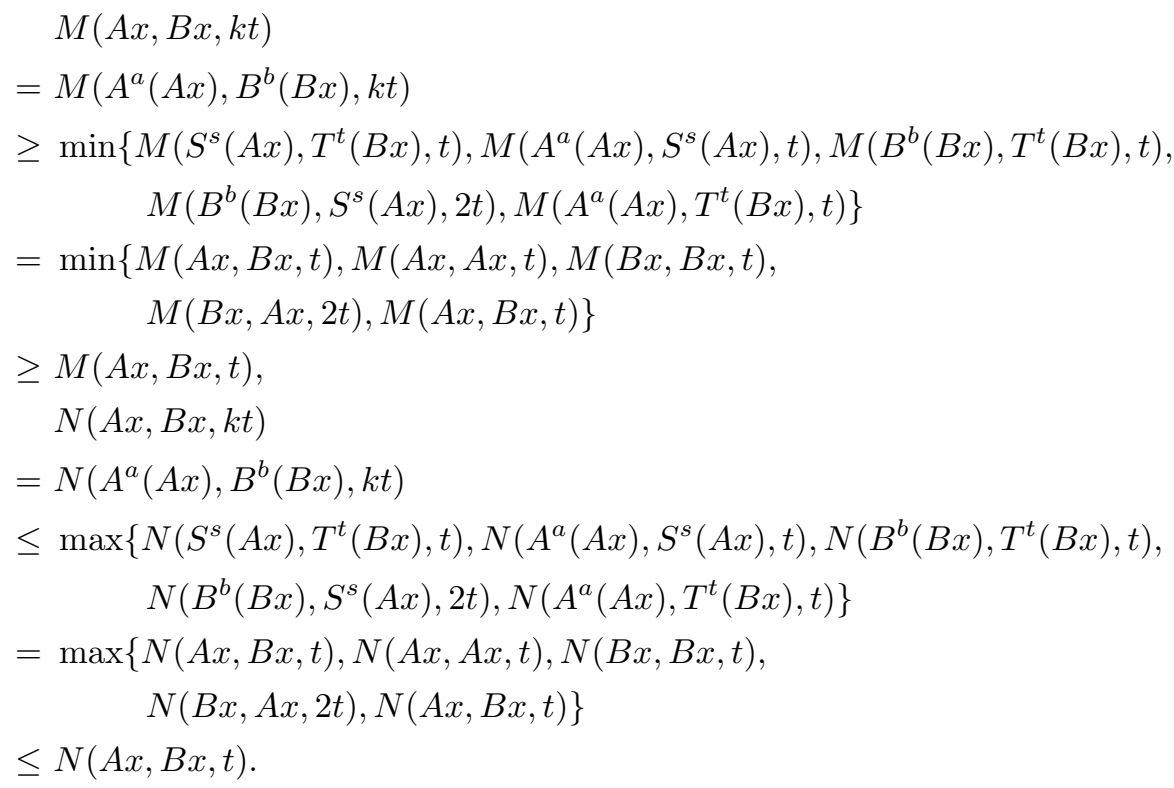

Therefore $A x=B x$. Also, we have

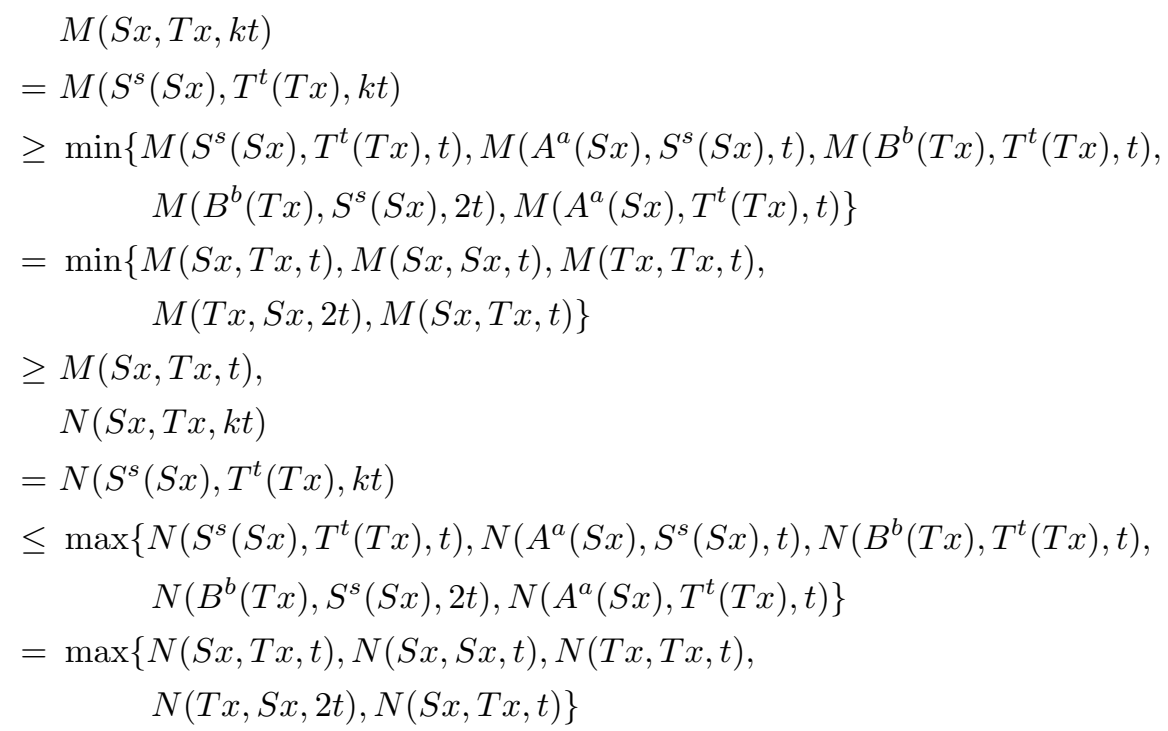




$$
\leq N(S x, T x, t) \text {. }
$$

Therefore $S x=T x$. Since $x$ is a unique common fixed point of $A^{a}, B^{b}, S^{s}, T^{t}$, hence $A x=B x$ is common fixed points of $A^{a}, S^{s}$ and $S x=T x$ is common fixed points of $B^{b}, T^{t}$. Hence $x=A x=B x=S x=T x$. That is, $x$ is a common fixed point of $A, B, S$ and $T$.

Corollary 3.2 ([14]). Let $A, B, S$ and $T$ be self maps of complete fuzzy metric space $(X, M, *)$ with continuous $t$-norm $*$ defined by $a * b=\min \{a, b\}, a, b \in$ $[0,1]$, satisfying the following conditions:

(a) $A(X) \subset T(X), B(X) \subset S(X)$.

(b) $S$ and $T$ are continuous.

(c) $(A, S),(B, T)$ are compatible pairs of maps.

(d) For all $x, y \in X, k \in(0,1), t>0$,

$$
\begin{aligned}
& M(A x, B y, k t) \\
& \geq \min \{M(S x, T y, t), M(A x, S x, t), M(B y, T y, t), \\
&M(B y, S x, 2 t), M(A x, T y, t)\} .
\end{aligned}
$$

(e) For all $x, y \in X, \lim _{t \rightarrow \infty} M(x, y, t)=1$.

Then $A, B, S$ and $T$ have a unique common fixed point in $X$.

Proof. For fuzzy metric space $X$, if $a=b=s=t=1$ in Condition (d) of Theorem 3.1, then the proof follows from Theorem 3.1.

\section{References}

[1] S. Banach, Theorie des operations lineaires, Monografje Mathematyczne., Warsaw 1932.

[2] M. Grabiec, Fixed point in fuzzy metric spaces, Fuzzy Sets and Systems 27 (1988), 385-389.

[3] G. Jungck, Compatible mappings and common fixed points, Internat. J. Math. Math. Sci. 9 (1986), 779-791.

[4] Compatible mappings and common fixed point (2), Internat. J. Math. Math. Sci. 11 (1988), no. 2, 285-288.

[5] J. Kramosil and J. Michalek, Fuzzy metric and statistical metric spaces, Kybernetica 11 (1975), 326-334.

[6] J. H. Park, Intuitionistic fuzzy metric spaces, Chaos Solitons \& Fractals 22 (2004), no. 5, 1039-1046.

[7] J. H. Park, J. S. Park, and Y. C. Kwun, A common fixed point theorem in the intuitionistic fuzzy metric space, Advances in Natural Comput. Data Mining(Proc. 2nd ICNC and 3rd FSKD) (2006), 293-300.

[8] J. S. Park, On some results in intuitionistic fuzzy metric space, J. Fixed Point Theory \& Appl. 3 (2008), no. 1, 39-48.

[9] J. S. Park and S. Y. Kim, A fixed point Theorem in a fuzzy metric space, F. J. M. S. 1 (1999), no .6, 927-934.

[10] Common fixed point theorem and example in intuitionistic fuzzy metric space, J. K. I. I. S. 18 (2008), no. 4, 524-529.

[11] J. S. Park and Y. C. Kwun, Some fixed point theorems in the intuitionistic fuzzy metric spaces, F. J. M. S. 24 (2007), no. 2, 227-239.

[12] J. S. Park, Y. C. Kwun, and J. H. Park, A fixed point theorem in the intuitionistic fuzzy metric spaces, F. J. M. S. 16 (2005), no. 2, 137-149. 
[13] B. Schweizer and A. Sklar, Statistical metric spaces, Pacific J. Math. 10 (1960), no. 10, $314-334$.

[14] B. Singh and M. S. Chauhan, Common fixed points of compatible maps in fuzzy metric spaces, Fuzzy Sets and Systems 115 (2000), 471-475.

[15] L. A. Zadeh, Fuzzy sets, Inform. and Control 8 (1965), 338-353.

Department of Mathematical Education Chinju National University of Education

JinJu 660-756, KorEA

E-mail address: parkjs@cue.ac.kr 